\section{El reto de la virtualidad en la educación odontológica}

\author{
The challenge of virtuality in \\ dental education
}

Alma Graciela García-Calderón 1,a, María Verónica Cuevas-González 1,b

${ }^{1}$ Universidad Autónoma de Ciudad Juárez, Instituto de Ciencias Biomédicas, Chihuahua, México.

a Odontóloga. Maestra en Docencia.

${ }^{b}$ Odontóloga. Doctora en Ciencias.

\section{Correspondencia:}

María Verónica Cuevas-González: maria.cuevas@uacj.mx

Av. Benjamín Franklin no. 4650, Zona Pronaf Condominio La Plata, 32310 Cd Juárez, Chihuahua. México.

ORCID: 0000-0003-3112-4893

Coautores:

Alma Graciela García-Calderón: alma.garcia@uacj.mx ORCID: 0000-0002-6297-3567

Recibido: 04/12/21

Aceptado: 09/12/21

Publicado: $21 / 01 / 22$
Debido a la situación sanitaria causada por el COVID-19 la educación odontológica ha enfrentado múltiples retos, pasando de lo presencial a lo virtual, esto ha sido todo un proceso, al ser una situación que se presentó de manera repentina las clases virtuales se tuvieron que implementar rápidamente, si bien la virtualidad era algo que ya se venia implementando en nuestro medio, es claro que esta pandemia aceleró su utilización y que en cierta manera hubo un cambio en la enseñanza que permanecerá después de que esta pandemia sea superada.

Entre las estrategias que las facultades y escuelas de odontología han implementado se encuentran: grabación de las clases teóricas, uso de las distintas plataformas para clase en línea que permita la interacción alumno-profesor, manejo de lecturas complementarias, realización de material didáctico, manejo de tutoriales, entre otros ${ }^{1}$.

Esta pandemia ha favorecido la implementación de nuevos modelos educativos los cuales buscan el equilibrio de actividades virtuales y semipresenciales ${ }^{2}$, distintos centros educativos han tomado la decisión de mantener las clases virtuales en aquellas materias teóricas y cuando las condiciones locales se los ha permitido y siguiendo adecuadas medidas de bioseguridad han favorecido la actividad presencial de las áreas con práctica clínica, las cuales son un grupo importante en el área de la estomatología.
Debido al distanciamiento social, el cual fue implementado de manera general por las autoridades sanitarias, la educación en línea ha sido la herramienta que ha permitido que las actividades académicas no se hayan interrumpido por completo, sobre todo al inicio de la emergencia sanitaria cuando la información que se tenia hasta ese momento era escasa, pero desenmascaró otra problemática, la cual es la falta de acceso a recursos tecnológicos, y a una adecuada conexión a internet ${ }^{3}$. Nuestra experiencia es similar a la reportada por estos autores, incluso algunos estudiantes nos refirieron no contar con las condiciones ni espacios adecuados en sus hogares para tomar las clases que favorecieran la concentración o les permitiera enfocarse a los contenidos temáticos, sin embargo, aun así esta situación se ha ido resolviendo de manera gradual, en algunos casos por parte de los alumnos y en el resto de los casos debido a la intervención de las autoridades universitarias al brindar en la medida de lo posible los espacios o medios electrónicos propicios.

Lo antes redactado ha representado un gran esfuerzo tanto para alumnos como docentes, los primeros al dedicar el tiempo y espacio adecuado para tomar las materias en cuestión, evitando en las clases en línea la distracción y cumplir con las actividades programadas y para los docentes adecuar los contenidos temáticos, actualización en tecnologías aplicadas en la educación y en general todo lo relacionado al desarrollo del curso ${ }^{4}$. 
En resumen si bien falta camino por avanzar antes de considerar como terminada esta pandemia, conforme va pasando el tiempo ha permitido ver las cosas con mayor claridad, conforme se vayan normalizando las actividades educativas seguramente se requerirán estrategias de evaluación para conocer el nivel de conocimiento con el que los alumnos están egresando de la licenciatura o las especialidades odontológicas y en su caso emplear estrategias para fortalecer las áreas solidas y trabajar en aquellas áreas de oportunidad que surjan.

\section{Referencias bibliográficas}

1. Tello-Espejo M, Castillo-Quispe E, Ochoa-Escalante E. Los retos de la educación virtual en odontología en tiempos de la pandemia de COVID-19. Rev Cubana Inv Bioméd. 2021;40:1497.
2. Inocente-Díaz ME, Díaz-Pizán ME. Educación superior dental: un reto para el docente universitario en tiempos de pandemia. Odontol Sanmarquina. 2020;23:215-218.

3. Guevara-Veliz DN, Flores-Joaquin KM, Maturrano-Santo AG, Mattos-Vela MA. Educación virtual en odontología durante la pandemia de COVID-19. Rev Cient Odontol (Lima). 2021;9:78.

4. Inciarte Rodriguez M. Competencias docentes ante la virtualidad de la educación superior. Télématique. 2008;7:19-38. 